\title{
The chemical composition of Local Group dwarf spheroidals
}

\author{
Eline Tolstoy \\ Kapteyn Institute, University of Groningen, Postbus 800, 9700AV Groningen, the Netherlands
}

\begin{abstract}
I will review the progress of VLT spectroscopy of large numbers of individual stars in nearby dwarf spheroidal galaxies. This spectroscopy has allowed us to obtain detailed insights into the chemical and dynamical properties of the resolved stellar population in these nearby systems.
\end{abstract}

\section{Introduction}

The dwarf galaxies being considered here are the lowest luminosity (and mass) galaxies that been have found. It is perhaps not a surprise that there is a lower limit in mass for an object to be able to form more than one generation of stars which will be related to the limit below which one supernova will completely destroy a galaxy. Numerical and analytic models tell us that the limit must be around a few $\times 10^{6} M_{\odot}$ (e.g., Ferrara \& Tolstoy 2000). Globular clusters, for example, typically have much lower masses than this limit.

It is among dwarf galaxies, irregular (dI) and spheroidal (dSph), that we find the lowest mass objects. For example the Sculptor dSph galaxy which apparently has a present day mass of $6 \times 10^{6} M_{\odot}$, or the dI galaxy DDO 210 which has a present day mass of $5.4 \times 10^{6} M_{\odot}$ (Mateo 1998). The only difference between the lowest mass dSphs and dIs seems to be the presence of gas and current star formation in dIs. It has already been noted that dSphs predominantly lie close to our galaxy $(<250 \mathrm{kpc}$ away), and dIs predominantly further away (>400 kpc away). This suggests that the proximity of dSph to our Galaxy played a role in the removal of gas from these systems. Although the range of properties found in dSph and dI galaxies does not allow a straight forward explanation; particularly not the large variations in star formation histories and chemical evolution paths that have now been observed in these systems.

Here I am going to discuss some recent observations of individual stars in nearby dSph galaxies, predominantly from the large survey being undertaken by DART (Dwarf Abundances and Radial-velocities Team) to measure abundances and velocities for several hundred individual stars in a sample of three nearby dSph galaxies: Sculptor, Fornax and Sextans. We have used the VLT/FLAMES facility to obtain more than 100 spectra per 25arcmin diameter field of view across several positions in each galaxy out to the tidal radius (see Figure 1). We have used the low resolution setting to obtain Ca II triplet metallicity estimates as well as accurate radial velocity measurements (Tolstoy et al. 2004; Battaglia et al., in prep) over a large area in each galaxy, and we have also used the high resolution settings to concentrate on about 100 stars in the central field of each galaxy - where we are able to obtain accurate abundances for a range of interesting elements such as Mg, Ca, O, Ti, Na, Eu to name a few (Hill et al., in prep; Letarte et al., in prep). 


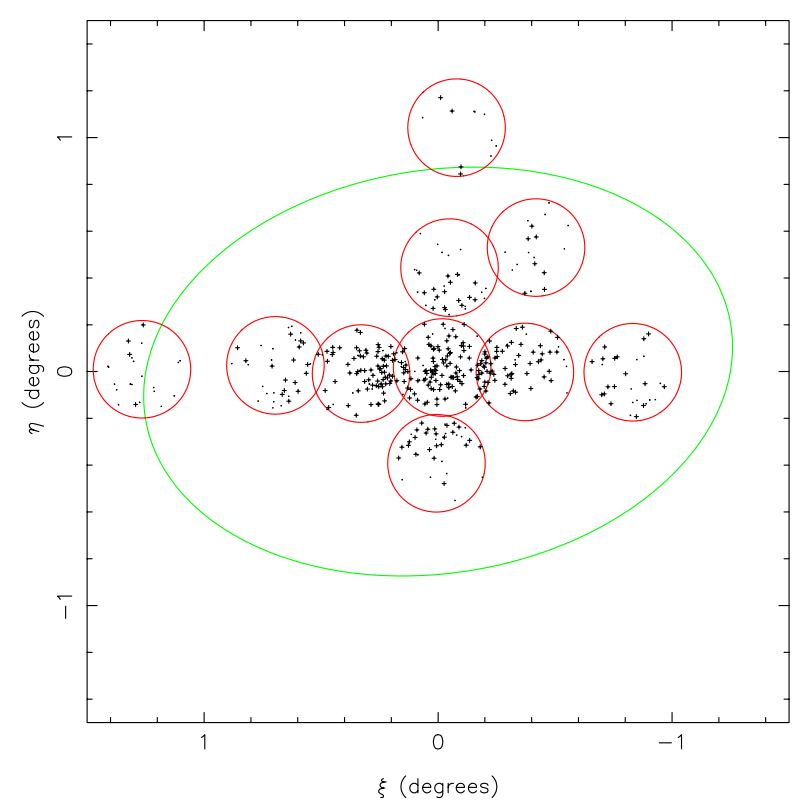

Figure 1. The distribution of candidate members RGB stars (shown as small crosses) and velocity non-members (dots) in the Sculptor dSph galaxy chosen from velocities measured in VLT/FLAMES spectra. The ellipse is the tidal radius of Sculptor, determined by Irwin \& Hatzidimitriou (1995). The circles represent the 10 individual FLAMES pointings made by DART in this galaxy.

\section{How do we understand the evolution of galaxies}

When we have resolved the individual stars in a galaxy and accurately measured their colours and magnitudes down to the oldest main sequence turn-offs $\left(\mathrm{M}_{V} \sim+3.5\right)$ in a Colour-Magnitude Diagram we can in principle come to detailed conclusions about how the star formation rate has varied with time all the way back to the first star formation in the galaxy (e.g., Tolstoy \& Saha 1996; Gallart et al. 1999; Hurley-Keller et al. 1999). This approach is the most accurate for intermediate age populations, but for stars older than about 10 Gyr the time resolution gets quite poor (and the stars are getting very faint), and it becomes hard to distinguish a 12 Gyr old star from a 10 Gyr old star. Here it becomes useful to consider the Horizontal Branch stars $\left(\mathrm{M}_{V} \sim 0\right.$.) which are the bright He-burning phase of low mass stars $>10$ Gyr old. The ratio of red to blue horizontal branch stars tells us about the age and metallicity variation (Lee et al. 2001) of the oldest stars in the galaxy (e.g., for Sculptor dSph, see Figs $2 \& 3$ ).

The Red Giant Branch (RGB, $-3<M_{V}<0$.) contains stars with ages $>1$ Gyr old, back to the oldest stars in the galaxy. In the majority of RGB stars it is believed that the atmosphere of the star remains an unpolluted sample of the interstellar medium out of which it was formed. This means we have small pockets of interstellar medium of different ages (enriched by different numbers of processes) conveniently covering the nuclear burning core of stars. This hot stellar core provides a useful bright background source to be absorbed in the stellar atmosphere allowing very detailed studies of the elemental abundances in these ancient gas samples. Thus, a spectroscopic analysis of the variation of the abundances of different elements seen in absorption in atmospheres of 


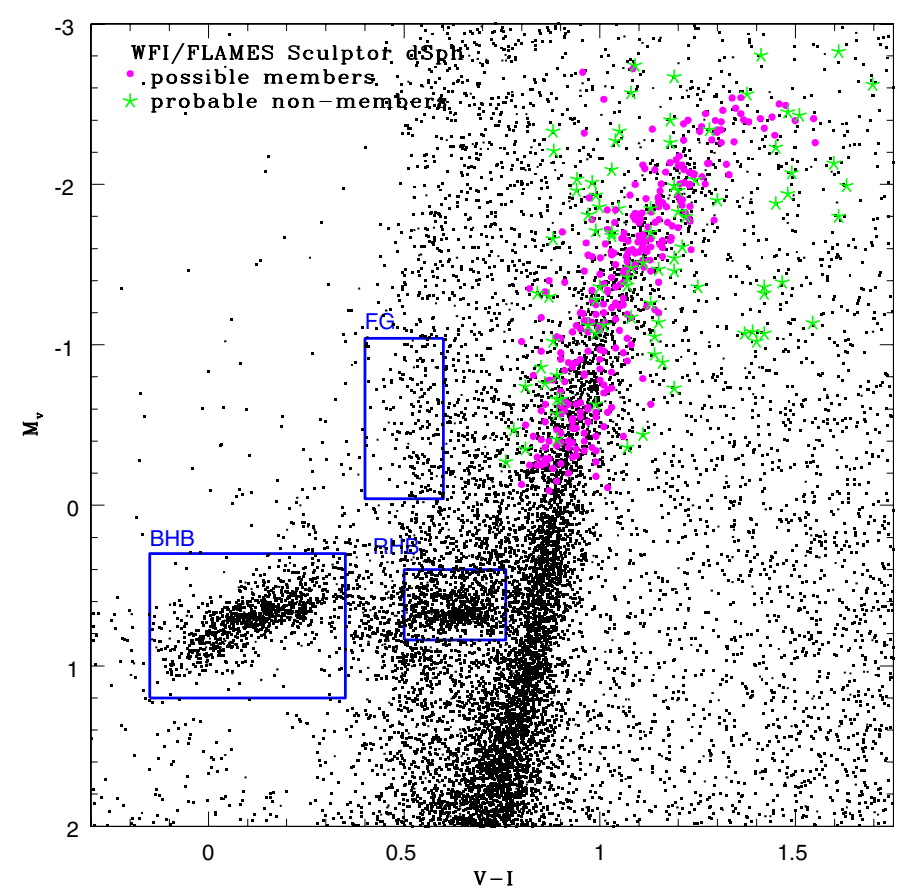

Figure 2. The Colour-Magnitude Diagram for the WFI coverage of Scl shown in Figure 1. The circle and star symbols are the Red Giant Branch stars which were observed with VLT/FLAMES and for which we have accurate $\mathrm{v}_{\text {hel }}$ and $[\mathrm{Fe} / \mathrm{H}]$ measurements $(\mathrm{S} / \mathrm{N}>10)$. The potential members of Scl are shown as circles and non-members shown as stars. Also shown are the regions used to define the Blue Horizontal branch (BHB), the red horizontal branch (RHB) and foreground comparison (FG) populations.

different age stars allows us to trace the detailed chemical enrichment history of a galaxy with time.

The age-metallicity degeneracy on the RGB (graphically illustrated by Cole et al. 2005; their Figure 8) leads to serious problems using photometric colour alone to disentangle age and metallicity. This uncertainty is the leading reason for quite different star formation histories to be inferred from the same Colour-Magnitude Diagrams. For example, in the case of the Carina $\mathrm{dSph}$ three groups determined three different star formation histories from two different data sets (Hurley-Keller et al. 1998; Hernandez et al. 2000; Dolphin 2002), some of the (significant) differences can be traced to different assumptions about the chemical evolution of Carina. However if similar chemical enrichment histories are assumed then the consistency between models by different groups is significantly improved (e.g., Skillman et al. 2003).

The observed magnitude and colour (e.g., $\mathrm{M}_{V}, \mathrm{~V}-\mathrm{I}$ ) of a star combined with a measured metallicity, $[\mathrm{Fe} / \mathrm{H}]$, allows us to effectively remove the age-metallicity degeneracy and determine the age of an RGB star from an isochrone. However the uncertainties in this age increase with decreasing metallicity, and often the photometric errors on the colour measurements are of the same order of magnitude as the entire age range of the isochrones from the oldest (13 Gyr) to the youngest (2 Gyr). This is before even considering other effects such as the variations in the Galactic reddening, $\alpha$-element abundance, distance, metallicity measurement error or errors in the interpolation of the models. 


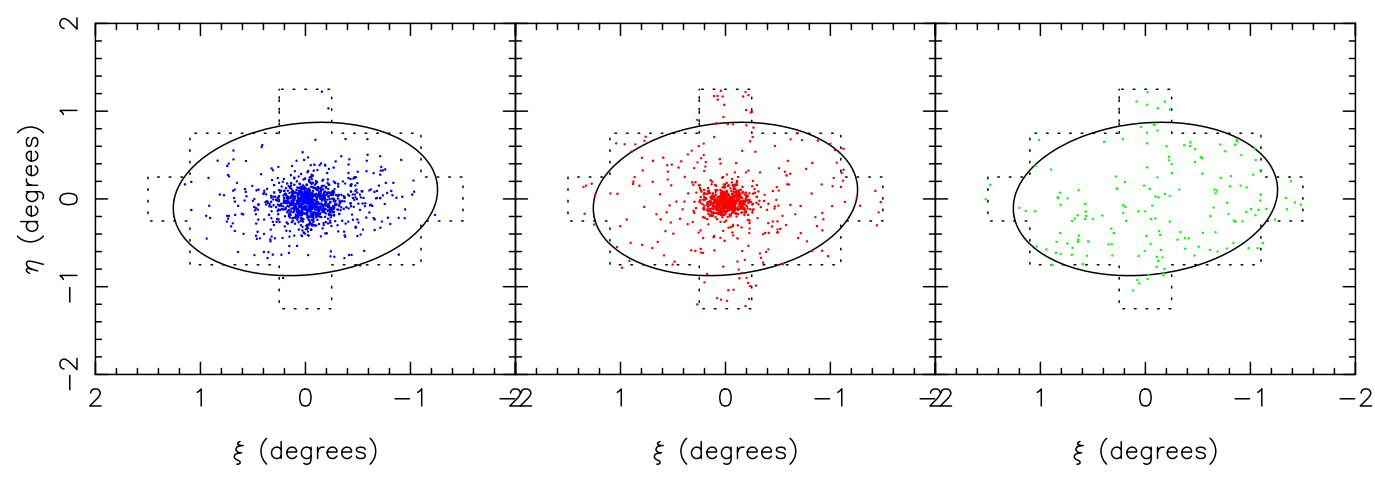

Figure 3. The distribution of Horizontal Branch stars from ESO/WFI imaging of the Scl dSph. The three panels show the different spatial distributions of blue horizontal branch stars (BHB), and red horizontal branch stars (RHB) as selected from the $\mathrm{M}_{v}, \mathrm{~V}-\mathrm{I}$ Colour-Magnitude Diagram (CMD) shown in Figure 2. Also shown, to illustrate the foreground contamination in the RHB distribution, are a CMD-selected sample of foreground stars to match the RHB contamination density (see Figure 2). The ellipse is the tidal radius of Scl as defined by Irwin \& Hatzidimitriou (1995).

The interpretation of all these stellar measurements rely upon stellar evolution models which are primarily based upon our understanding of Galactic globular cluster stars and we must make the assumption that this knowledge is generally applicable to stars in dwarf galaxies even though the initial abundance ratios are different, and certainly the stellar density is very different.

\section{High Resolution Spectroscopy: Detailed Abundance Analysis}

The best way to measure the chemical evolution of a galaxy is to determine the relative abundances of different chemical elements. This has been called chemical tagging by Freeman \& Bland-Hawthorn (2002). Different elements are created in different circumstances and if we are able to determine the abundance of elements known to be created in a particular set of physical conditions, we can assess the importance and frequency of these conditions during the history of star formation in a galaxy. This clearly can give insights into the star formation rates at different times in a galaxy's history and the environments in which most of the stars were formed.

For example, the abundance of light elements (e.g., O, Na, Mg, Al) are considered to be tracers of "deep mixing" patterns which are found only in globular cluster environments, which gives a limit to the number of dissolved globular clusters which can exist in a stellar population. These typical (Galactic) globular cluster abundance patterns have also been recently found in the globular clusters of Fornax dSph (Letarte et al. 2005), but not (so far) in the field star populations (Shetrone et al. 2003).

The creation of $\alpha$-elements (e.g., O, Mg, Si, Ca, Ti) occurs predominately in Supernovae type II explosions, i.e. the explosion of massive stars a few $10^{6}-10^{7}$ yrs after their formation. The abundance of the different $\alpha$-elements is quite sensitive to the mass of the SNII progenitor (e.g., Woosley \& Weaver 1995) so the $\alpha /$ Fe ratio traces the mass function of the stars which contributed to the creation of the $\alpha$ elements, and ratios of different $\alpha$ elements themselves can put limits on the highest mass star which has enriched a galaxy and also the typical mass range (e.g., McWilliam 1997). 


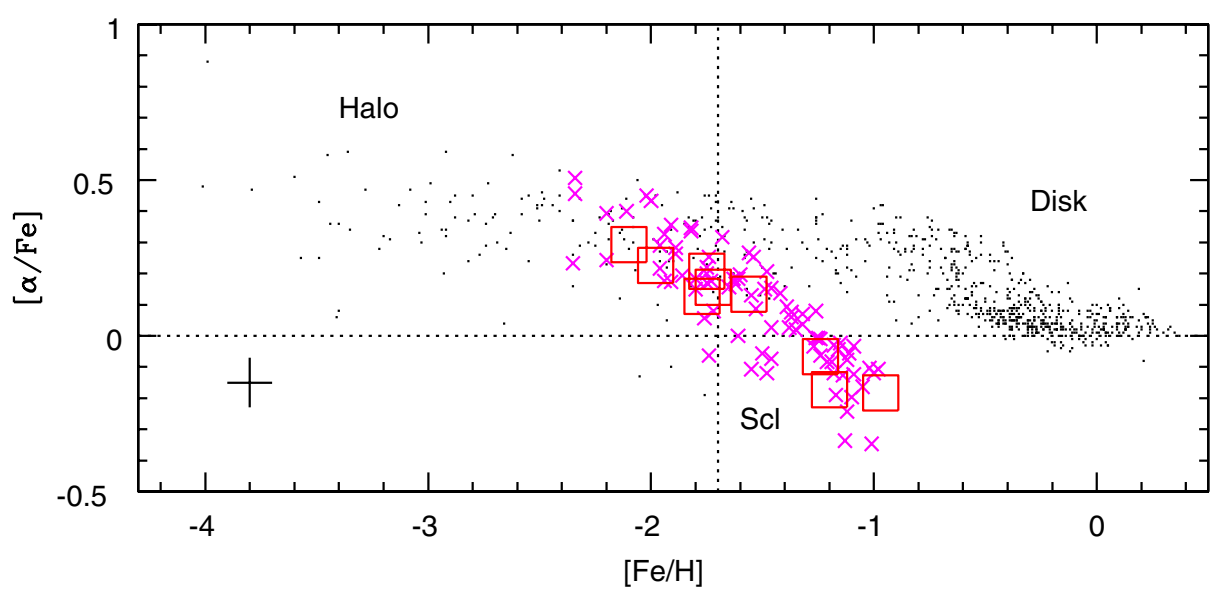

Figure 4. The $\alpha$-abundance (average of $\mathrm{Ca}, \mathrm{Mg}$ and $\mathrm{Ti}$ ) for stars in our Galaxy compared to those in Scl. The VLT/FLAMES high resolution measurements of 92 members in the central field are shown as crosses (from Hill et al., in prep). The Galactic stars come from standard literature sources (see Venn et al. 2004 for references). The 10 open squares are UVES measurements of individual stars in Scl (Shetrone et al. 2003; Geisler et al. 2005).

Heavy Elements $(\mathrm{Z}>30)$ are a mix of $\mathrm{r}$ - and $\mathrm{s}$ - process elements. That is to say elements which were produced by rapid or slow neutron capture which tells us about the environment in which enrichment occurred. Rapid capture (r-process) is assumed to occur in high energy circumstances, such as supernovae explosions. For example Eu is considered to be an element produced almost exclusively by the r-process. The slow capture (s-process) is thought to be created by more quiescent processes such as the stellar winds common in AGB type stars of intermediate age (and mass). Typical elements which are thought to be created by the s-process are Ba and La. The ratio between $\mathrm{r}-$ and s-process element abundances gives an indication of the relative importance of these different enrichment processes during the history of star formation in a galaxy. Dwarf spheroidal galaxies are generally found to have strong s-process enhancement. This may indicate that supernovae products are typically lost to a shallow potential well, or that the slow star formation rate means that massive stars are not very common (Tolstoy et al. 2003; Venn et al. 2004).

With an $8 \mathrm{~m}$ class telescope it is possible to measure the abundance of these elements in dSph galaxies in a few hours of integration time with a high-resolution high-throughput spectrograph, e.g. KECK/HIRES (Shetrone et al. 2001); VLT/UVES (Tolstoy et al. 2003; Shetrone et al. 2003; Geisler et al. 2005; Letarte et al. 2005, in prep). But it has been quite slow and laborious to obtain a statistically meaningful sample of stars in dSph galaxies which have fairly complicated star formation histories. This complex star forming history means that a sizeable sample of stars of different ages is required to trace the full range enrichment processes. The real break-through comes with VLT/FLAMES (and other fibre systems, such as MIKE on Magellan). In one shot spectra can be obtained for over 100 stars in one 25 arcmin field of view. The sensitivity and the field of view are a very nice match to the Galactic dSph galaxies.

The results published to date still lack good statistics to make a detailed comparison between our Milky Way and individual dSph, although attempts have been made (e.g, Tolstoy et al. 2003; Venn et al. 2004). From these results, and the early results from our FLAMES surveys (e.g., Hill et al., in prep; Battaglia et al., in prep) a match is not 
found between the detailed abundance properties of stellar populations found in dSph galaxies and any (significant) component of our Galaxy. There remains a discrepancy between the metallicity $([\mathrm{Fe} / \mathrm{H}])$ distribution of the stars randomly selected in a dSph (e.g., Scl: Tolstoy et al. 2004; also true for Fornax and Sextans, Battaglia et al. in prep) and samples of stars in our Milky Way. This is also true when more detailed comparisons are made, for example considering how $[\alpha / \mathrm{Fe}]$ varies with $[\mathrm{Fe} / \mathrm{H}]$ (Hill et al. 2004 , in prep; see Figure 4).
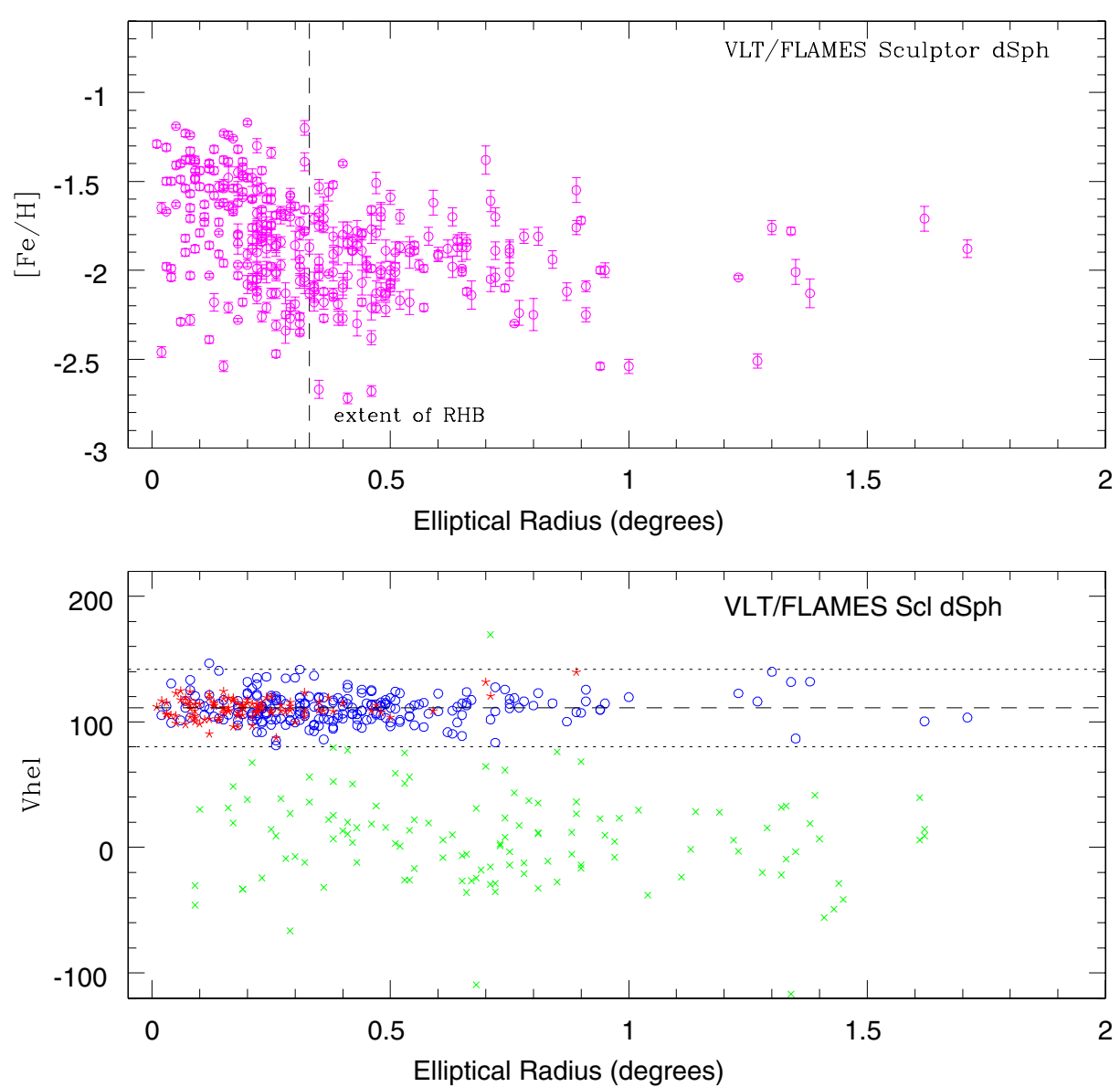

Figure 5. The upper panel shows the VLT/FLAMES spectroscopic measurements of $[\mathrm{Fe} / \mathrm{H}]$ for the 321 potential RGB velocity members of $\mathrm{Scl}$ (and $\mathrm{S} / \mathrm{N}>10$ ). We see a clear trend of metallicity with radius. The lower panel shows $\mathrm{v}_{h e l}$ as a function of elliptical radius for all stars satisfying S/N > 10. Likely Scl members are clearly seen clustered around the systemic velocity of $110 \mathrm{~km} / \mathrm{s}$. The 321 stars which are potential members are plotted as stars $([\mathrm{Fe} / \mathrm{H}]>-1.7)$ and circles $([\mathrm{Fe} / \mathrm{H}]<-1.7)$, while the 128 crosses are assumed to be non-members.

\section{Low Resolution Spectroscopy: Metallicities}

The ideal is to be able to obtain high resolution spectra for individual stars in nearby dSph over a large wavelength range, and make a detailed analysis of a range of different elements. However, this is quite time consuming both in telescope time (at the 
distance of dSph) and in analysis. One of the most simple ways to get an estimate of the metallicity of RGB stars is with the Ca II triplet (e.g., Cole et al. 2004). This is a basic metallicity indicator requiring only low or intermediate spectral resolution, based on three lines around $8500 \AA$ A which have been empirically calibrated from observations of stars in globular clusters stars with high resolution abundances (e.g., Armandroff \& Da Costa 1991).

With the Ca II triplet we obtain an overview of the global metallicity range of the RGB stars in a dwarf galaxy. In the case of the DART project these measurements are complementary to the high resolution observations made in the centre of each dSph. In the low resolution larger area survey we can assess how representative our detailed study is of the stellar population of the whole galaxy.

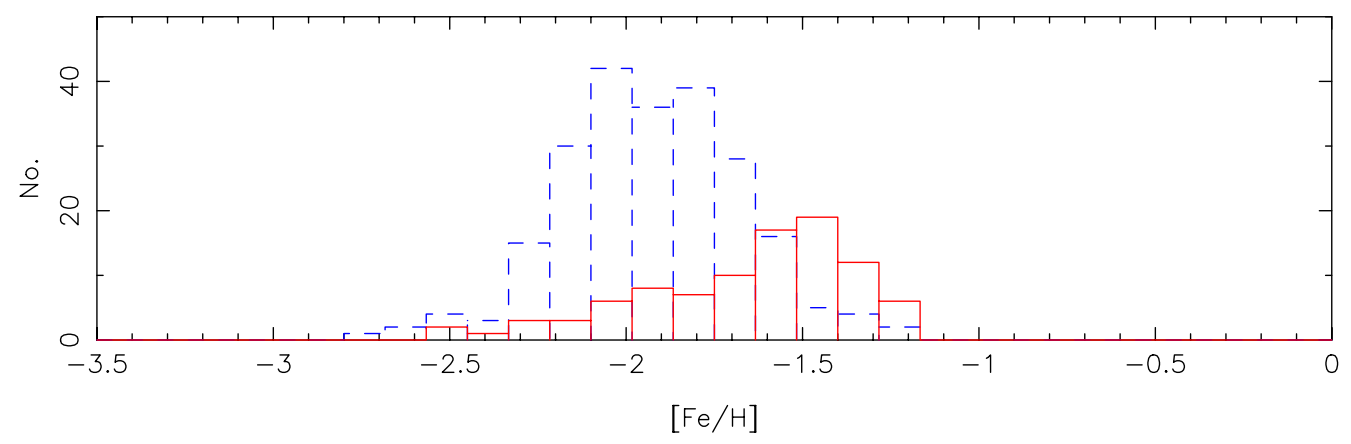

Figure 6. Here is the histogram distribution of $[\mathrm{Fe} / \mathrm{H}]$ for $\mathrm{Scl}$ dSph: the 94 stars within the central, $\mathrm{r}<0.2$ degree region (solid line); and the 227 stars beyond $\mathrm{r}>0.2$ degrees (dashed line).

Our first VLT/FLAMES results, based upon Ca II triplet, clearly show that Scl dSph contains two distinct stellar components with different spatial, kinematic and abundance properties (Tolstoy et al. 2004, see Figure 5). There appears to be a metal-rich, $-0.9>$ $[\mathrm{Fe} / \mathrm{H}]>-1.7$, and a metal-poor, $-1.7>[\mathrm{Fe} / \mathrm{H}]>-2.8$ component. The metal-rich component is more centrally concentrated than the metal poor, and on average appears to have a lower velocity dispersion, $\sigma_{\text {metal-rich }}=7 \pm 1 \mathrm{~km} / \mathrm{s}$, whereas $\sigma_{\text {metal-poor }}=$ $11 \pm 1 \mathrm{~km} / \mathrm{s}$.

The full abundance analysis of the FLAMES HR data (Hill et al. in prep) will provide more details of the chemical enrichment history of Scl. This will enable us to distinguish between two episodes of star formation or more continuous star formation, manifested as a gradient in velocity dispersion and metallicity from the centre of the galaxy.

It is clear from the histogram of $[\mathrm{Fe} / \mathrm{H}]$ measurements in Scl dSph (see Figure 6) that this distribution lacks a low metallicity tail, in fact the lowest metallicity star in our sample of more than 300 stars is $[\mathrm{Fe} / \mathrm{H}]=-2.7$. We find a similar lack of low metallicity stars in Fornax and Sextans. Although it is difficult to make an accurate comparison with Galactic samples, where the completeness of different samples is hard to quantify, there appears to be a significantly different distribution between dSph and the (metal-poor) halo of the Milky Way.

\section{Conclusions}

There are indications that the presence of two populations is a common feature of dSph galaxies. Our preliminary analysis of Horizontal Branch stars, vhel and $[\mathrm{Fe} / \mathrm{H}]$ measurements in the other galaxies in our sample (Fornax and Sextans dSph; Battaglia et al., in prep) also show similar characteristics to Scl, especially in the most metal 
poor component. Pure radial velocity studies (Wilkinson et al. 2004; Kleyna et al. 2004) have also considered the possibility that kinematically distinct components exist in Ursa Minor, Draco and Sextans dSph galaxies.

What mechanism could create two ancient stellar components in a small dwarf spheroidal galaxy? A simple possibility is that the formation of these dSph galaxies began with an initial burst of star formation, resulting in a stellar population with a mean $[\mathrm{Fe} / \mathrm{H}] \leqslant-2$. Subsequent supernovae explosions from this initial episode could have been sufficient to cause gas (and metal) loss such that star formation was inhibited until the remaining gas could sink deeper into the centre (e.g., Mori et al. 2002). Thus the subsequent generation(s) of stars would inhabit a region closer to the centre of the galaxy, and have a higher average metallicity and different kinematics. Another possible cause is external influences, such as minor mergers, accretion of additional gas or the kinematic stirring by our Galaxy. It might also be that events surrounding the epoch of reionisation influenced the evolution of these small galaxies (e.g., Skillman et al. 2003) and resulted in the stripping or photo-evaporation of the outer layers of gas in the dSph, meaning that subsequent more metal enhanced star formation occurred only in the central regions.

\section{Acknowledgements}

I am grateful for support from a fellowship of the Royal Netherlands Academy of Arts and Sciences, and the exceptional collaborators that make up DART, especially Mike Irwin, Giuseppina Battaglia, Amina Helmi, Andrew Cole, Vanessa Hill, Kim Venn, Bruno Letarte, Pascale Jablonka, Matthew Shetrone \& Andreas Kaufer.

\section{References}

Armandroff, T. \& Da Costa, G.S. 1991, AJ 101, 1329

Cole A.A., Smecker-Hane T.A., Tolstoy E. et al. 2004, MNRAS 347, 367

Cole A.A., Tolstoy E., Gallagher J.S. \& Smecker-Hane T.A. 2005, AJ 129, 1465

Dolphin A. 2002, MNRAS 332, 91

Ferrara A. \& Tolstoy E. 2000, MNRAS 313, 291

Freeman K. \& Bland-Hawthorn J. 2002, ARA\&A 40, 487

Gallart C., Freedman W.L., Aparicio A., Bertelli G. \& Chiosi C. 1999, AJ 118, 2245

Geisler, D., Smith, V., Wallerstein, G., Gonzalez, G. \& Charbonnel, C. 2005, AJ 129, 1428

Hernandez X., Gilmore, G. \& Valls-Gabaud, D. 2000, MNRAS 317, 8831

Hurley-Keller D., Mateo M., \& Nemec J. 1998, AJ 115, 1840

Hurley-Keller D., Mateo M., \& Grebel E.K. 1999, ApJL 523, 25

Irwin M. \& Hatzidimitriou D. 1995, MNRAS 277, 1354

Kleyna J.T., Wilkinson M.I., Evans N.W. \& Gilmore G. 2004, MNRAS 354, L66

Lee Y.-W. et al. 2001 in: T. von Hippel et al. (eds.) Astrophysical Ages and Time Scales, ASP

Conference Series, vol. 245, p. 343

Mateo M. 1998, ARA\&A 36, 435

McWilliam A. 1997, ARA\&A 35, 503

Mori M., Ferrara A. \& Madau P. 2002, ApJ 571, 40

Shetrone M.D., Cote P. \& Sargent W.L.W. 2001, ApJ 548, 592

Shetrone M.D., Venn K.A., Tolstoy E., Primas F., Hill V. \& Kaufer A. 2003, AJ 125, 684

Skillman E.D., Tolstoy E., Cole A.A., Dolphin A.E. et al. 2003, ApJ 596, 253

Tolstoy E. \& Saha A. 1996, ApJ 462, 672

Tolstoy E., Venn K.A., Shetrone M.D., Primas F., Hill V. et al. 2003, AJ 125, 707

Tolstoy E., Irwin M.J., Helmi A., Battaglia G., Jablonka P., Hill V. et al. 2004, ApJL 617, 119

Venn K., Irwin M., Shetrone M.D., Tout C.A., Hill V. \& Tolstoy E. 2004, AJ 128, 1177

Wilkinson M., Kleyna J.T., Evans N.W., Gilmore G. et al. 2004, ApJL 611, 21 